\title{
Hepatitis and the polyglandular autoimmune syndrome, type 1
}

Jolanta Białkowska ${ }^{1}$, Arkadiusz Zygmunt², Andrzej Lewiński², Wanda Stankiewicz ${ }^{3}$, Alina Knopik-Dąbrowicz', Wojciech Szubert ${ }^{5}$, Maciej Jabłkowski ${ }^{1}$

${ }^{1}$ Department of Infectious and Liver Diseases, Medical University of Lodz, Poland 2Department of Endocrinology and Metabolic Diseases, Medical University of Lodz, Poland

${ }^{3}$ Microwave Safety Department, Military Institute of Hygiene and Epidemiology, Warsaw, Poland

4Bieganski Hospital, Lodz, Poland

${ }^{5}$ Department of Radiology, Medical University of Lodz, Poland

Submitted: 17 June 2010

Accepted: 14 October 2010

Ach Med Sci 2011; 7, 3: 536-539

DOI: 10.5114/aoms.2011.23427

Copyright (c) 2011 Termedia \& Banach

\begin{abstract}
Autoimmune polyglandular syndrome, type 1 (APS-1) is a rare syndrome. Here we present a case report of a 24-year-old female patient who complained of progressive weakness. While autoimmune hepatitis was diagnosed, no improvement of biochemical parameters was obtained after immunosuppressive treatment. Hypoparathyroidism and adrenocortical failure were identified. Her health status clearly improved once proper control of the calcium-phosphate metabolism was obtained and after the administration of substitution hydrocortisone doses, leading to full normalization of biochemical liver tests. The reported case illustrates a rare form of APS-1 failure, in which the diagnosed autoimmune hepatitis was only the first symptom.
\end{abstract}

Key words: hepatitis, polyglandular autoimmune syndrome.

\section{Introduction}

Autoimmune polyglandular syndrome, type 1 (APS-1, also known as Whitaker's syndrome, Blizzard's syndrome and autoimmune polyglandularcandidiasis-ectodermal dystrophy syndrome - APECEDS) is a rare syndrome of autoimmune, multi-organ disorders, together with blastomycosis and ectodermal dystrophy [1, 2]. Autoimmune polyglandular syndrome, type 1 results from mutations in the autoimmune regulator gene (AIRE) at locus 21q22.3, which encodes a 54-kDa protein that is present in the epithelial cells of the thymus core and participates in control of central and peripheral immunological tolerance $[3,4]$. The diagnosis of APS-1 is based on identification of, at least two, out of the three, most frequently observed components of the syndrome (recurring candidosis, hypoparathyroidism, and adrenal cortex failure); however, other components may also occur, including type 1 diabetes mellitus, hypergonadotropic hypogonadism, vitiligo, and alopecia areata [5]. The clinical picture may include diarrhoea, symptoms of poor absorption resulting from the atrophy of endocrine cells in the gastric tract and dysfunction of parietal cells that may lead to pernicious anaemia. Additional symptoms may include enamel hypoplasia,

\author{
Corresponding author: \\ Maciej Jabłkowski MD, PhD \\ Department of Infectious \\ and Liver Diseases \\ Medical University of Lodz \\ 1/5 Kniaziewicza \\ Lodz, Poland \\ Phone: +48 422516299 \\ Fax: +48 422516006 \\ e-mail: \\ m.jablkowski@pro.onet.pl
}


nail dystrophy, tympanic membrane sclerosis, and acquired leukoderma. In some patients, congenital asplenia, cholelithiasis (likely resulting from absorption disorders), or autoimmune hepatitis may be observed.

\section{Case report}

A 24-year-old non-smoking woman was admitted to the Department of Infectious Diseases, Medical University of Lodz, Poland, for progressive weakness and elevated aminotransferase activities that persisted over 3 months: ALT up to $300 \mathrm{U} / \mathrm{l}$ and AST up to $250 \mathrm{U} / \mathrm{l}$.

The patient's history included cholecystectomy, performed 2 years before for cholelithiasis and subcapsular cataract surgery in the right eye with artificial lens implantation (surgery date not available). Recurring candidosis of the oral cavity and hypoparathyroidism had been observed since the patient's early childhood. Unfortunately, the patient had not been covered by continuous endocrine care. The patient used nystatin in topical applications for the recurring candidosis; however, the patient did not receive calcium, vitamin $D$, or its metabolites for hypoparathyroidism. She did not receive any other medications, and she had never consumed alcohol. She regularly menstruated, did not use any hormonal contraception, and was not pregnant.

No deviations from normal status were found in the medical examination performed upon admission. Abdominal sonography revealed a normalsized liver with uniform echostructure, a typical post-cholecystectomy status. Laboratory blood serum tests provided the following results: ALT 508 $\mathrm{U} / \mathrm{l}$, AST $324 \mathrm{U} / \mathrm{l}$, activity of $\gamma$-glutamyl-transpeptidase $15 \mathrm{U} / \mathrm{l}$, alkaline phosphatase $197 \mathrm{U} / \mathrm{l}$, total bilirubin concentration $1.55 \mathrm{mg} / \mathrm{dl}$, total protein $8.51 \mathrm{~g} / \mathrm{dl}$, and $\gamma$-globulin 30\% (2.55 g/dl). Blood cell count, electrolytes, urea, and creatinine test results were all within normal values.
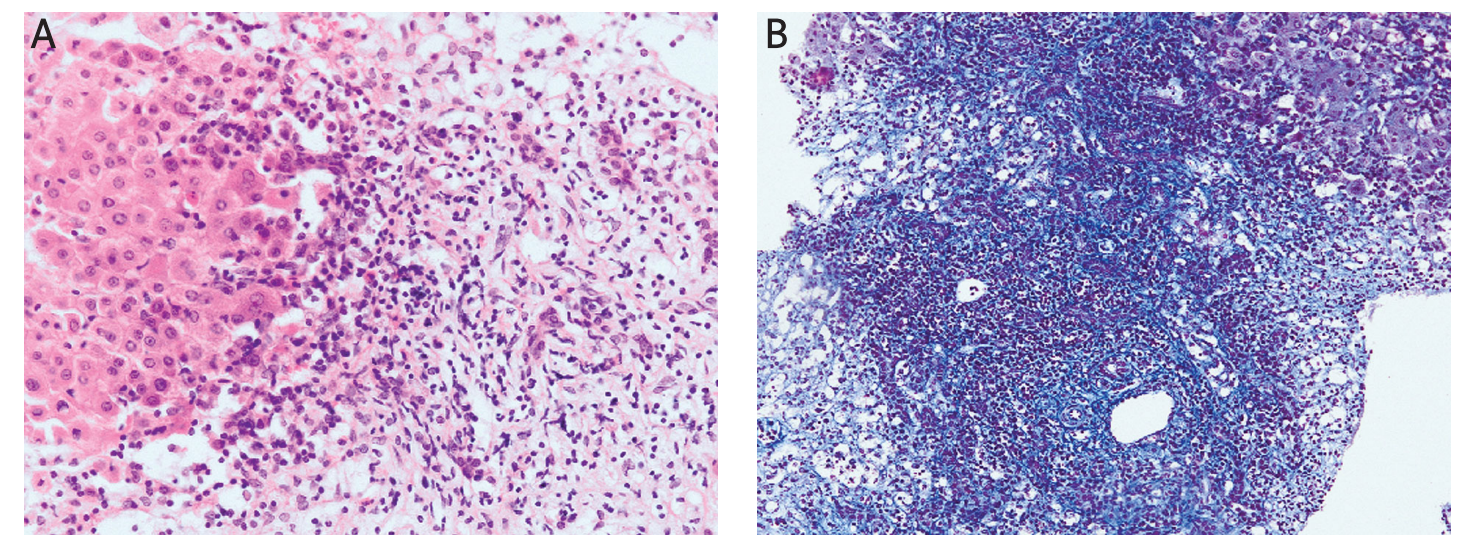

Figure 1. A. Abundant inflammatory infiltrations from lymphoid and plasmatic cells in portal-biliary spaces, necrosis of hepatocytes on peripheral hepatic lobules (piecemeal necrosis type). B. Hyperplasia of connective-fibrous tissue within portal spaces. Porto-portal bridges sporadically visible

Antinuclear antibodies were identified at $113.8 \mathrm{lU} / \mathrm{ml}(\mathrm{N}>23$ positive result). No other antibodies were detected, including antimitochondrial antibodies, type 1 liver and kidney anti-microsomal antibodies, anti-soluble liver antigen antibodies, and anti-liver cytosol antibodies. Hepatitis A, B, and C viruses, as well as Wilson's disease, were excluded.

High aminotransferase activities were observed during hospitalization. After 3 months, high activities of ALT (353 U/I) and AST (230 U/I) persisted, while measured thyroid-stimulating hormone, $\mathrm{FT}_{3}$, and $\mathrm{FT}_{4}$ concentrations were within normal values. Histopathology of a collected liver section revealed chronic hepatitis of moderate activity with inflammatory infiltrations of lymphoid and plasmatic cells and with periportal and bridging porto-portal fibrosis. This microscopic image corresponded to autoimmune hepatitis (Figures 1 A, 1 B).

Type 1 autoimmune hepatitis was diagnosed in the patient (the value of the Autoimmune Hepatitis Scoring System was 17), prompting the administration of immunosuppressive therapy (prednisolone $30 \mathrm{mg} /$ day). Improvements, such as decreased ALT (116 U/l) and AST (69 U/l), were achieved, as was a reduction in $\gamma$-globulins $(1.7 \mathrm{~g} / \mathrm{dl})$. Azathioprine was then added at a dose of $100 \mathrm{mg} /$ day, with the prednisolone dose reduced to $5 \mathrm{mg} /$ day. After 2 months of the therapy, repeated rises in ALT values were noted (up to $211 \mathrm{U} / \mathrm{l}$ ) and in AST values (up to $86 \mathrm{U} / \mathrm{l}$ ). During a subsequent hospitalisation, tetany attack occurred in the patient for the first time in her life, with no deviations from the normal values in neurological examinations (data not shown).

Computed tomography (CT) of the head was then performed. In its initial phase, CT imaging revealed hyperdense foci, corresponding to massive calcifications in the nuclei of the base of the brain, in the thalamus, as well as subcortical in the white matter of both frontal lobes (Figure 2). Massive calcifications are also visible in the nuclei and in the 


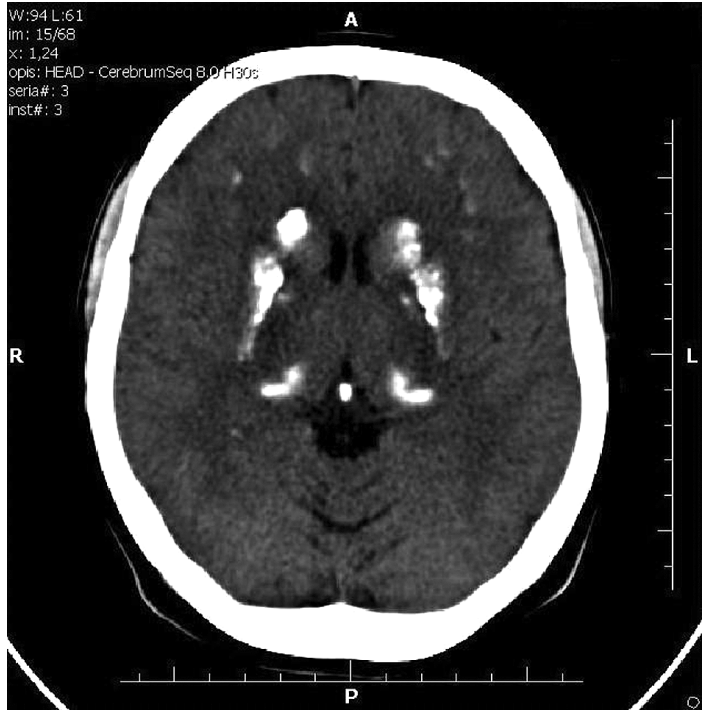

Figure 2. Computed tomography imaging revealed hyperdense foci, corresponding to massive calcifications in the nuclei of the base of the brain, in the thymuses, as well as subcortical in the white matter of both frontal lobes

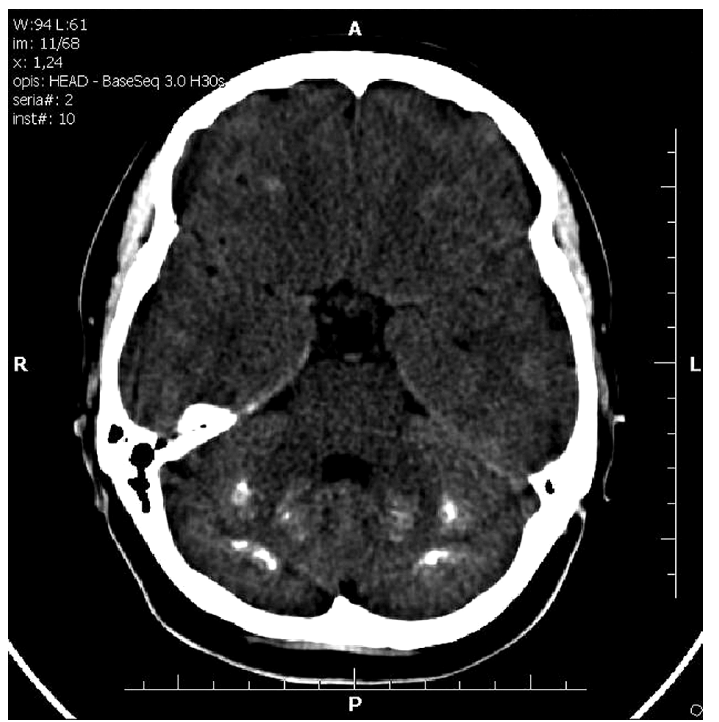

Figure 3. Massive calcifications were visible in the nuclei and in the white matter of both cerebellar hemispheres

white matter of both cerebellar hemispheres (Figure 3). The changes were symmetrically configured in both cerebral and cerebellar hemispheres.

No foci of pathological strengthening were identified during the second phase of CT imaging after contrast administration (data not shown).

Laboratory studies revealed a considerable decrease in the total serum calcium concentration (corrected vs. albumin concentration) to $3.5 \mathrm{mg} / \mathrm{dl}$ (normal: $8.8-10.4 \mathrm{mg} / \mathrm{dl}$ ), together with a high phosphorus concentration of $12.3 \mathrm{mg} / \mathrm{dl}$ (normal: 2.8-4.5 mg/dl) and a decreased parathormone concentration of $5.78 \mathrm{pg} / \mathrm{ml}$. Due to identified calcium-phosphate metabolism disorders, the patient was transferred to the Clinic of Endocrinology and Metabolic Diseases.

The recurring oral candidosis and the primary hypoparathyroidism prompted the diagnosis of APS-1. The third, main component of the syndrome is primary adrenocortical failure; no symptoms of adrenal cortex failure were found in the patient, while electrolyte concentration values and glycaemia were normal. Five weeks after withdrawal of the suppressive glucocorticosteroid doses, an adrenal cortex stimulation test with corticotrophin (250 $\mu \mathrm{g}$ Synacthen administered intravenously) was performed. Cortisol concentration at the $60^{\text {th }}$ min of the test was $11.9 \mu \mathrm{g} / \mathrm{dl}$, indicating the presence of primary adrenocortical failure. The autoimmunological basis for the occurrence of primary adrenocortical failure was confirmed by the increased titre of anti-adrenal cortex antibodies ( $1: 20$ vs. the normal value of $1: 10$ ).

The applied treatment was multidirectional, with a concomitant focus on dietary education targeted at reducing the volumes of consumed phosphates. Further treatment was launched, including calcium agents $\left(\mathrm{CaCO}_{3}\right)$, and vitamin $\mathrm{D}$, with doses selected to maximally normalize the calcium-phosphate metabolism parameters. The goal of the therapy was to achieve calcium concentration in serum oscillating around the lower normal limit. The patient was informed about adrenocortical failure and adrenocortical crisis prognostic symptoms. Hydrocortisone substitution was launched, emphasizing the importance of flexible dose modifications, taking into account the concomitant stress.

After 12 months of therapy, the patient currently feels well and does not report any complaints. The most recent laboratory tests returned the following results: serum ALT $14 \mathrm{U} / \mathrm{l}$, AST $18 \mathrm{U} / \mathrm{l}$, total bilirubin $0.67 \mathrm{mg} / \mathrm{dl}$, total calcium $7.8 \mathrm{mg} / \mathrm{dl}$, and phosphorus $4.52 \mathrm{mg} / \mathrm{dl}$.

\section{Discussion}

Autoimmune hepatitis $(\mathrm{AlH})$ is a chronic, progressive, necrotic-inflammatory process in the liver of unknown aetiology. Advancing lesions of hepatic parenchyma may lead to liver cirrhosis [6] and even to acute liver failure [7]. Three types of $\mathrm{AlH}$ have been described, based on immunoserological findings. Here we have presented a case of autoimmune hepatitis as the initial symptom of APS-1. The diagnosis of autoimmune hepatitis was made from the criteria of the International Autoimmune Hepatitis Scoring System in Brighton, and the International Autoimmune Hepatitis Group $[8,9]$. High ALT, AST, and $\gamma$-globulin activities were found in blood serum, the presence of anti-nuclear antibodies was confirmed, and features of 
inflammation and periportal fibrosis with inflammatory infiltrations from lymphoid and plasmatic cells were seen in the histopathology of a collected liver biopsy.

The patient received typical anti-suppressive treatment with steroids, completed by addition of azathioprine [10]. While a reduction of aminotransferase activities was initially achieved, the levels increased after 3 months. Only the unique attack of tetany prompted the extension of the diagnostic protocol.

Fungal infections (Candida) are a frequently described clinical symptom of APS-1, affecting the mucosal membranes of the oral cavity, the oesophagus, and distal sections of the gastric tract, as well as the skin and its appendages [11]. The immune background for chronic candidosis is a selective deficit of $T$ lymphocytes and the associated impaired response to the presence of antigens. While it is true that the patient did report recurring episodes of oral candidosis in her history, experienced since early childhood, the infections were slight and easily explained by the administered therapy with anti-fungal agents, and thus they did not arouse anxiety.

A previous report has described the case of an untreated 10-year-old girl who exhibited APS-1 symptoms from the $3^{\text {rd }}$ year of her life, including chronic nail and oral candidosis and hypoparathyroidism [12]. At the $6^{\text {th }}$ year of the child's life, skin xanthochromia occurred, which then spontaneously regressed. Four years later, symptoms of hepatic failure developed, and despite urgent liver transplantation, the child died a few years after the operation. Advanced hepatitis with massive necrosis was the histopathological diagnosis made after evaluation of a collected liver section.

Another report presents data from a 31-year observational study of 68 patients with APS-1 [13]. Features of chronic hepatitis were identified in 12 of the patients, while in one patient diagnosed autoimmune hepatitis was the first symptom of APS-1. Acute hepatic failure was the direct cause of death in two out of nine deceased patients. Autoimmune hepatitis occurs in $15-18 \%$ of APS-1 patients, sometimes directly causing death.

In conclusion, when patients with autoimmune hepatitis are unresponsive to standard immunosuppressive treatment, concomitant hormone metabolism disorders should be taken into consideration.

\section{References}

1. Eisenbarth GS, Gottlieb PA. Autoimmune polyendocrine syndromes. N Engl I Med 2004; 350: 2068-79.

2. Kahaly GJ. Polyglandular autoimmune syndromes. Eur J Endocrinol 2009; 161: 11-20.
3. Su MA, Giang K, Zumer K, et al. Mechanisms of an autoimmunity syndrome in mice caused by a dominant mutation in Aire. J Clin Invest 2008; 118: 1712-26.

4. DeVoss JJ, Shum AK, Johannes KP, et al. Effector mechanisms of the autoimmune syndrome in the murine model of autoimmune polyglandular syndrome type 1 . J Immunol 2008; 181: 4072-9.

5. Betterle C, Greggiio NA, Volpato M. Autoimmune polyglandular syndrome type 1. J Clin Endocrin Metab 1998; 83: 1049-55.

6. Feld J, Dinh H, Arenovich T, Marcus VA, Wanless IR, Heathcote EJ. Autoimmune hepatitis: effect of symptoms and cirrhosis on natural history and outcome. Hepatology 2005; 42: 53-62.

7. Krawitt EL. Autoimmune hepatitis. N Engl J Med 2006; 354: 54-66.

8. Alvarez F, Berg PA, Bianchi FB, et al. International autoimmune hepatits group report: review of criteria for diagnosis of autoimmune hepatitis. J Hepatol 1999; 31: 929-38.

9. Vergani D, Alvarez F, Bianchi FB, et al. Liver autoimmune serology: a consensus statement from the committee for autoimmune serology of the International Autoimmune Hepatitis Group. J Hepatol 2004; 41: 677-83.

10. Manns MP, Vogel A. Autoimmune hepatitis, from mechanisms to therapy. Hepatology 2006; 43 (2 Suppl. 1): 132-44.

11. Peterson P, Perheentupa J, Krohn KJ. Detection of Candidal antigens in autoimmune polyglandular syndrome type I. Clin Diagn Lab Immunol 1996; 3: 290-4.

12. Michele TM, Fleckenstein J, Sgrignoli AR, Thyluvath PJ. Chronic active hepatitis in the type I polyglandular autoimmune syndrome. Postgrad Med J 1994; 70: 128-31.

13. Ahonen P, Myllärniemi S, Sipilä I, Perheentupa J. Clinical variation of autoimmune polyendocrinopathy-candidiasisectodermal dystrophy (APECED) in a series of 68 patients. N Eng J Med 1990; 322: 1829-36. 